\title{
Internal-illumination photoacoustic computed tomography
}

\author{
Mucong Li \\ Bangxin Lan \\ Wei Liu \\ Jun Xia \\ Junjie Yao
}




\section{Internal-illumination photoacoustic computed tomography}

\author{
Mucong Li, ${ }^{a}$ Bangxin Lan, ${ }^{a}$ Wei Liu, ${ }^{a}$ Jun Xia, ${ }^{b}$ and \\ Junjie Yao ${ }^{\mathrm{a}, *}$ \\ aDuke University, Department of Biomedical Engineering, Durham, \\ North Carolina, United States \\ bUniversity at Buffalo North Campus, Department of Biomedical \\ Engineering, Buffalo, New York, United States
}

\begin{abstract}
We report a photoacoustic computed tomography (PACT) system using a customized optical fiber with a cylindrical diffuser to internally illuminate deep targets. The traditional external light illumination in PACT usually limits the penetration depth to a few centimeters from the tissue surface, mainly due to strong optical attenuation along the light propagation path from the outside in. By contrast, internal light illumination, with external ultrasound detection, can potentially detect much deeper targets. Different from previous internal illumination PACT implementations using forward-looking optical fibers, our internal-illumination PACT system uses a customized optical fiber with a 3-cm-long conoid needle diffuser attached to the fiber tip, which can homogeneously illuminate the surrounding space and substantially enlarge the field of view. We characterized the internal illumination distribution and PACT system performance. We performed tissue phantom and in vivo animal studies to further demonstrate the superior imaging depth using internal illumination over external illumination. We imaged a 7.5-cm-deep leaf target embedded in optically scattering medium and the beating heart of a mouse overlaid with 3.7-cm-thick chicken tissue. Our results have collectively demonstrated that the internal light illumination combined with external ultrasound detection might be a useful strategy to improve the penetration depth of PACT in imaging deep organs of large animals and humans. () 2018 Society of Photo-Optical Instrumentation Engineers (SPIE) [DOI: 10.1117/1.JBO.23.3.030506]
\end{abstract}

Keywords: photoacoustic imaging; photoacoustic computed tomography; internal illumination; fiber diffuser; deep imaging.

Paper 170813LR received Dec. 19, 2017; accepted for publication Mar. 5, 2018; published online Mar. 23, 2018.

\section{Introduction}

Photoacoustic imaging (PAI) is a hybrid imaging modality that combines optical excitation with ultrasound detection. ${ }^{1}$ Compared to pure optical imaging such as confocal ${ }^{2,3}$ and two photon microscopy, ${ }^{3,4}$ PAI is less limited by optical diffusion for deep tissue imaging, taking advantage of both ballistic and diffused photons. In PAI, the deposited photon energy (scattered or not) is converted to acoustic waves, which are scattered much less than photons in biological tissues, and thus PAI can penetrate deeper while maintaining acoustically determined spatial resolutions. ${ }^{1,5}$ Moreover, different from ultrasound imaging, the signals in PAI originate from optical absorption rather than acoustic back reflection, providing inherent sensitivity to tissue's functional status and molecular composition, ${ }^{6}$ with various endogenous and exogenous contrast agents.

Among different implementations of PAI, photoacoustic computed tomography (PACT) ${ }^{7-12}$ is the most promising for clinical translation, due to its deep penetration and high compatibility with commercial ultrasound imaging systems. In PACT, the laser pulses are usually directed to the tissue surface by using a multimode optical guide, which is often bifurcated to flank the transducer array and forms a dark-field optical illumination pattern on the tissue surface. ${ }^{1,13}$ Yet due to the strong attenuation of photons (e.g., effective attenuation coefficient of human breast tissue is $\sim 4.0 \mathrm{~cm}^{-1}$ at $730 \mathrm{~nm}^{14}$ ), it remains a great challenge for PAT to penetrate beyond a few centimeters. The largest penetration in PAT reported so far is about $11 \mathrm{~cm}$ in chicken breast tissue using highly absorbing exogenous contrast agents. ${ }^{1,15}$

In many clinical situations, the region of interest can be deeply seated underneath the skin surface but relatively close to some body cavities. For example, the prostate can be more than $10 \mathrm{~cm}$ from the abdominal surface but is only 2 to $3 \mathrm{~cm}$ from the nearby rectal cavity. Therefore, it is possible to image the deepseated organs by delivering light from inside through the body cavities. Thus, some internal-illumination PAI designs have emerged to tackle the penetration issue by embedding an optical fiber in the tissue and delivering light adjacent to the region of interest. The applications so far include identification of nerves, ${ }^{16,17}$ ex vivo human placenta imaging, ${ }^{18}$ and in vivo mouse brain imaging. ${ }^{19}$ In these systems, the photoacoustic waves are detected from the outside, and minimally invasive or noninvasive procedures are performed by exploiting body cavities such as the esophagus, the nasal cavity, and the rectum. ${ }^{17,18,20-22}$ However, the previous internal illumination designs rely on light radiating either from the bare fiber tip or from a small slot on the side of the fiber jacket, which cannot homogeneously illuminate the surrounding tissue. In addition, none of the aforementioned designs have been validated for imaging targets in vivo at depths of more than $5 \mathrm{~cm}^{21}$

In this study, we aim to achieve a larger photoacoustic imaging depth by employing a different internal illumination scheme. Compared with previous designs, the tip of the optical fiber used in our study was fused to a needle-shaped silica diffuser, which enables uniform illumination of a large tissue volume. Both tissue phantom and in vivo experiments were performed on deep targets using internal illumination, and the results were compared with those using external illumination. Our results have demonstrated that the new internal illumination with a fiber diffuser can improve the imaging depth of PACT.

\section{Methods}

The PACT system is based on a commercial ultrasound scanner (Vantage 128, Verasonics, Washington) with a linear array transducer (L7-4, 128 elements, $5 \mathrm{MHz}$ central frequency, $60 \%$ bandwidth) [Fig. 1(a)]. Laser pulses (pulsewidth: $8 \mathrm{~ns}$; Q-smart 850, Quantel Laser, Montana) are delivered through a customized multimode fiber (Pioneer Optics, Connecticut) with a numerical aperture (NA) of 0.43 and a core diameter of $1.5 \mathrm{~mm}$ [Fig. 1(b)]. The distal end of the optical fiber is fused with a 3-cm-long 


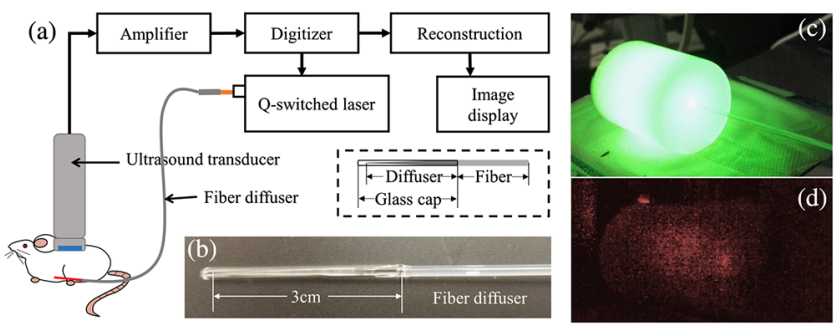

Fig. 1 Internal-illumination PACT with a customized fiber diffuser (a) Schematics of the PACT system. (b) The photograph of the fiber diffuser tip, showing the 3-cm-long cylindrical diffuser fused onto the fiber tip. (c) Fiber diffuser illumination pattern at $532 \mathrm{~nm}$. The scattering phantom was made of agar mixed with $0.5 \%$ intralipid. (d) Fiber diffuser illumination pattern at $1064 \mathrm{~nm}$. Note the image contrast is limited by the CCD camera's low sensitivity to the $1064 \mathrm{~nm}$ light.

active diffuser made of silica (diameter: $1.5 \mathrm{~mm}$ ). The entire diffuser is sealed by a transparent quartz cap with a diameter of $2.4 \mathrm{~mm}$. The function of the glass cap is twofold: (1) it protects the diffuser from contacting the tissue surface and thus avoids the fiber damage due to the "hot spots" stained by blood; (2) it traps air around the diffuser surface so that the light can propagate inside the entire diffuser due to the large refractive index mismatch between silica ( 1.5) and air (1.0), and gradually leak out along the diffuser to provide uniform cylindrical illumination pattern. Without the quartz cap, the diffuser surface can get in touch with the tissue or the body liquid, which has a similar refractive index $(\sim 1.37)$ with the silica. In this case, photons would leak out of the diffuser quickly without providing a cylindrical-shaped illumination pattern. Finally, two-dimensional PA data are acquired using the ultrasound scanner, and the offline image reconstruction is based on a traditional delay and sum method after correcting the transducer's receiving directivity.

We first investigated the illumination pattern of the fiber diffuser at both 532 and $1064 \mathrm{~nm}$. The fiber was inserted into an agarose (Sigma-Aldrich, Missouri) phantom mixed with $0.5 \%$ (v/v) intralipid to mimic the tissue scattering (Sigma-Aldrich, Missouri). The reduced scattering coefficient of the phantom was $\sim 7 \mathrm{~cm}^{-1}$ at $532 \mathrm{~nm}^{23,24}$ A CMOS camera was used to capture the illumination at both wavelengths. Then, we performed phantom experiments to characterize the PACT system with internal illumination. For the spatial resolution, a human hair with a diameter of $\sim 100 \mu \mathrm{m}$ was used as a line source. The spatial resolutions were quantified by calculating the full-width-athalf-maximum values of the hair signal along the axial and lateral directions in the image plane. To determine the penetration depth, we imaged a plastic tube (diameter: $2 \mathrm{~mm}$ ) filled with whole bovine blood. We overlaid an agarose phantom (thickness: $\sim 8 \mathrm{~cm}$ ) mixed with $0.5 \%(\mathrm{v} / \mathrm{v})$ fat milk (reduced scattering coefficient: $20 \mathrm{~cm}^{-1}$ at $750 \mathrm{~nm}^{25}$ ) above the hair or the tube. Another agarose phantom of the same composition (thickness: 15 to $50 \mathrm{~mm}$ ) was put beneath the target and above the fiber diffuser. Thickness of the bottom phantom was varied to alter the distance between the illumination source and the target. The imaging depth here is limited mostly by the light attenuation from the fiber diffuser to the target.

Deep imaging with internal illumination was demonstrated by performing both phantom and in vivo experiments. In the phantom experiment, a 7.5 -cm-thick agarose phantom mixed with $0.5 \%$ intralipid was placed above a red leaf skeleton to mimic blood vasculature. The fiber diffuser was placed beneath the leaf with a 1-cm-thick scattering phantom in between. The ultrasound transducer was mounted above the phantom with the image plane aligned with the fiber diffuser. Three-dimensional (3-D) data sets were acquired by translating the transducer via a stepper motor stage (VT-80, Micos Inc.), with a $150-\mu$ m step size. To compare the penetration depth with external illumination, a fiber bundle (Model number 54-203, Edmund Optics Inc., New Jersey) was mounted on the ultrasound transducer via a 3-D-printed adapter to illuminate phantom from the top.

In vivo experiments were conducted on a female Swiss Webster mouse (10 weeks old and $31.5 \mathrm{~g}$ in weight). The protocol was approved by the Institutional Animal Care and Use Committee of Duke University. The hair on the mouse's back and abdomen was removed. To mimic the future application of internal illumination on large animals, we overlaid 37mm-thick chicken breast tissue on top of the mouse's back. On top of the chicken tissue was a water tank that coupled the ultrasound transducer. Ultrasound gel was used to couple the tissue surface and the water tank bottom. The fiber diffuser was placed beneath the mouse abdomen. Laser pulses at $1064 \mathrm{~nm}$ (pulse energy: $\sim 30 \mathrm{~mJ}$ ) were delivered to the diffuser. The ultrasound transducer's image plane was near the mouse heart. A similar procedure was implemented using external illumination with the same output pulse energy, and the result was compared with that using internal illumination. To further demonstrate the penetration depth of the fiber diffuser, chicken breast tissue $(\sim 1 \mathrm{~cm}$ thick) was placed between the mouse abdomen and the fiber diffuser. Another 55-mm-thick chicken breast tissue was put between the mouse back and the ultrasound transducer. The same optical energy was applied to the mouse at $1064 \mathrm{~nm}$. A total number of 300 frames of PA images were acquired and averaged to improve the signal-to-noise ratio (SNR).

\section{Results and Discussion}

The illumination pattern of the fiber diffuser was investigated with both 532 and $1064 \mathrm{~nm}$ light. The entire agar phantom was uniformly illuminated as shown in Figs. 1(c) and 1(d), showing the homogeneity of light distribution at both wavelengths. The homogeneous illumination is critical for imaging a relatively large volume without adjusting the fiber position to point directly at the targets.

The PACT's resolutions were measured at an $\sim 80 \mathrm{~mm}$ depth. This depth is likely to be clinically relevant in the future (e.g., transrectal imaging of prostate cancers). The measured axial resolution is $419 \mu \mathrm{m}$ and the measured lateral resolution is $931 \mu \mathrm{m}$ [Figs. 2(a)-2(c)]. The measured resolutions are slightly worse than the theoretical values, mainly because the acoustic attenuation over the $80 \mathrm{~mm}$ distance between the target and the ultrasound transducer reduced the high frequency components of the PA signals and the effective NA of the beam forming.

The penetration depth was measured at $1064 \mathrm{~nm}$ with various target-to-fiber distances from 15 to $45 \mathrm{~mm}$ [Fig. 2(d)]. The SNR as a function of depth is shown in Fig. 2(e). The SNR decreased as the target moved away from the diffuser, with a decay rate of $0.65 \mathrm{~dB} / \mathrm{mm}$. These results have demonstrated that the internal illumination can provide an imaging depth of at least $45 \mathrm{~mm}$ from the internal fiber diffuser and $80 \mathrm{~mm}$ from the external ultrasound transducer.

A red leaf was imaged at a depth of $\sim 75 \mathrm{~mm}$ in the agar-intralipid phantom [Figs. 3(a)-3(c)]. The PA signals were acquired with both the internal illumination and external illumination. Three-hundred B-scans along the elevational direction were 

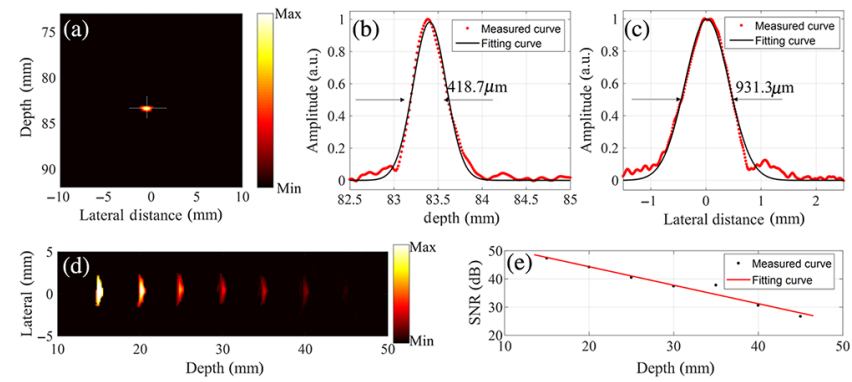

Fig. 2 Characterization of the internal illumination PACT system. (a) PACT image of a human hair at a depth of $\sim 83 \mathrm{~mm}$. (b) Axial image profile of the hair along the vertical white line in (a). (c) Lateral image profile of the hair along the horizontal white line in (a). (d) PACT images of a plastic tube filled with whole bovine blood embedded in a $0.5 \%$ fat milk phantom with $1064 \mathrm{~nm}$ light. The lateral axis represents the distance between the fiber diffuser and the blood tube. (e) Measured and fitted (polynomial model) SNR changes of blood signals as a function of depth at $1064 \mathrm{~nm}$.
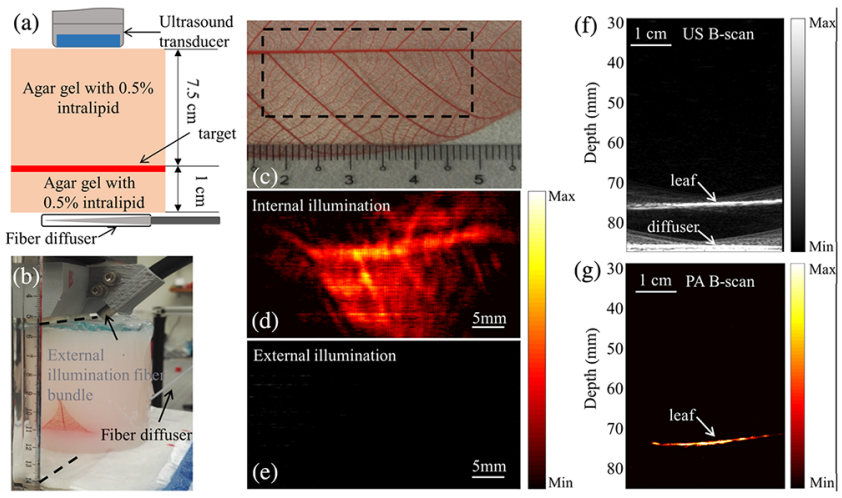

Fig. 3 Internal-illumination PACT of a leaf phantom. (a) Experimental schematics of the leaf phantom. The leaf is $7.5 \mathrm{~cm}$ from the ultrasound transducer on the top and $1 \mathrm{~cm}$ from the fiber diffuser at the bottom. (b) Photograph of the experiment setup. Two black dotted lines represent the top and bottom of the phantom. (c) Photograph of the leaf imaged by PACT. The area inside the black rectangle is the region of interest. (d-e) The PACT MAP images of the target using internal and external illumination, respectively. The vertical direction is the motor scanning direction. The same scale is used in both figures. $(\mathrm{f}-\mathrm{g})$ Ultrasound and PACT B-scan images of one cross section of the leaf target, respectively.

reconstructed, and the maximum amplitude projection (MAP) images along the axial direction were extracted. With the internal illumination, the leaf can be clearly imaged, as shown in Fig. 3(d). The PA image agrees well with the true leaf structure. By contrast, no PA signals were detected from the same area with the external illumination [Fig. 3(e)], mainly because most photons were attenuated within the first a few centimeters in the phantom. In contrast, with the internal illumination, photons did not have to travel a long distance to reach the target, and the effective penetration depth was improved. Figures 3(f) and $3(\mathrm{~g})$ show the ultrasound and photoacoustic B-scan images of the leaf, which agree well with each other.

To further demonstrate the deep imaging capability, in vivo studies were performed on a mouse overlaid with $37 \mathrm{~mm}$ chicken breast tissue. B-mode ultrasound imaging together with PACT with internal illumination were performed, as shown in
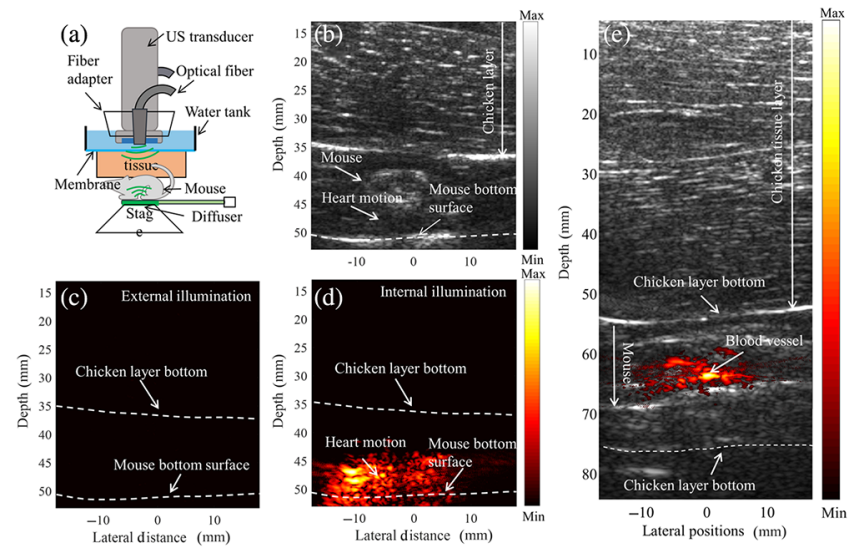

Fig. 4 Internal-illumination PACT of a mouse in vivo. (a) Experimental setup showing the mouse overlaid with chicken tissue on top. (b) The B-mode ultrasound image of a mouse cross-section overlaid with 3.7$\mathrm{cm}$-thick chicken breast tissue. Video 1 shows dynamic US imaging of the mouse cross-section overlaid with $3.7-\mathrm{cm}$-thick chicken breast tissue (gray color: Video 1, MP4, $141 \mathrm{~KB}$ ) [URL: https://doi.org/10.1117/ 1.JBO.23.3.030506.1]). (c-d) PACT B-scan images with (c) external and (d) internal illumination. Video 2 shows dynamic internal-illumination PACT of the mouse cross-section overlaid with $3.7-\mathrm{cm}$-thick chicken breast tissue (red color: Video 2, MP4, 59 KB [URL: https://doi.org/10.1117/1.JBO.23.3.030506.2]). (e) Merged PACT and US image of the mouse abdominal cross section with $1-\mathrm{cm}$ thick chicken breast tissue underneath and $5.5 \mathrm{~cm}$ on top.
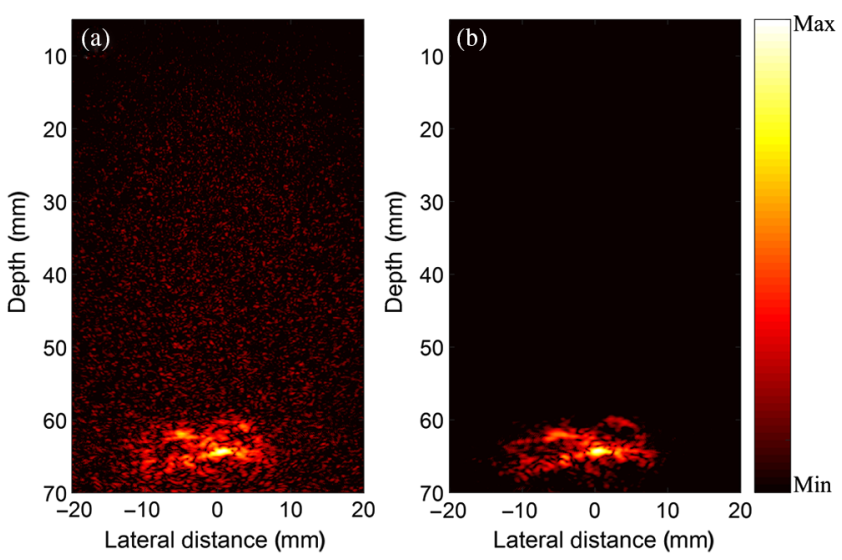

Fig. 5 Internal-illumination PACT images of the mouse acquired with (a) a single-laser-shot and (b) averaged with 300-laser shots. The same color scale applies to both images.

Fig. 4(a). The B-mode ultrasound imaging [Fig. 4(b) and Video 1] clearly shows the mouse overlaid with chicken breast tissue, and the tissue displacement caused by the breathing and heart beating was observed. While no PA signals were detectable with external illumination [Fig. 4(c)], the PA signals at $47 \mathrm{~mm}$ deep were detected with internal illumination with an SNR of $\sim 42 \mathrm{~dB}$ [Fig. 4(d) and Video 2]. Figure 4(e) further shows the penetration depth with internal illumination enabled by the fiber diffuser. Blood vessels in the mouse abdomen, which are $1.5 \mathrm{~cm}$ away from the fiber diffuser and $6.5 \mathrm{~cm}$ from the transducer, can be imaged with an SNR of $\sim 38 \mathrm{~dB}$. To compare the image quality, we have shown the singlelaser-shot image in Fig. 5(a) and the 300-times-averaged image in Fig. 5(b). 


\section{Conclusion}

In this letter, we present a new PACT scheme that illuminates the targets from inside with a fiber diffuser. Compared with the traditional external illumination, the internal illumination shows superior performance in imaging deep-seated targets in both phantom and in vivo studies. Compared with other interventional PACT methods using internal light illumination, our cylindrical fiber diffuser can potentially irradiate a larger volume of tissue and provide a more homogeneous illumination. Therefore, we believe that the internal illumination method can be useful for imaging internal organs that are deeply seated in the human body but close to the body cavity nearby, including the prostate close to the rectal cavity, the bladder, and kidney close to the urethra and ureter, and the lung close to the trachea. The imaging depth is less limited by the strong optical attenuation from the skin surface. However, the ultimate penetration depth is still limited by the optical attenuation from the fiber diffuser to the target of interest.

Several steps will be taken to further improve the image quality. The light energy delivered to the target is limited by the damage threshold of the fiber (peak power: 23.6 MW). A fiber diffuser with a larger core will be customized to deliver more energy and provide homogeneous illumination to a larger volume of tissue. Alternatively, there are cases in which it is not necessary to illuminate all the tissues around the fiber tip, so a fiber diffuser that can direct the light toward a particular direction may be utilized to improve the SNR. For example, a semicylindrical fiber tip with the flat surface coated with silver can reflect light only through the curved surface. With the optimized fiber diffuser, we expect the internal illumination PACT to find well-matched applications in clinical practice, especially for cancer screening and intraoperative guidance.

\section{Disclosures}

All authors have no financial interest.

\section{Acknowledgments}

This work was supported by Duke MEDx fund (to J. Yao). We thank Professors Kathy Nightingale and Gregg Trahey for their generous support of the Verasonics System, Ron Hille from Pioneer Optics Company for providing the fiber diffuser, and Emelina Vienneau for editing the manuscript.

\section{References}

1. L. V. Wang and S. Hu, "Photoacoustic tomography: in vivo imaging from organelles to organs," Science 335(6075), 1458-1462 (2012).

2. M. Rajadhyaksha et al., "In-vivo confocal scanning laser microscopy of human skin-melanin provides strong contrast," J. Invest. Dermatol. 104(6), 946-952 (1995).

3. E. Kahn et al., "Fluorescence excitation analysis by two-photon confocal laser scanning microscopy: a new method to identify fluorescent nanoparticles on histological tissue sections," Int. J. Nanomed. 7, 5545-5554 (2012).

4. W. Denk, J. H. Strickler, and W. W. Webb, "Two-photon laser scanning fluorescence microscopy," Science 248(4951), 73-76 (1990).

5. L. V. Wang, "Multiscale photoacoustic microscopy and computed tomography," Nat. Photonics 3(9), 503-509 (2009).

6. J. Yao and L. V. Wang, "Sensitivity of photoacoustic microscopy," Photoacoustics 2(2), 87-101 (2014).

7. R. A. Kruger et al., "Thermoacoustic computed tomography using a conventional linear transducer array," Med. Phys. 30(5), 856-860 (2003).

8. L. G. Montilla et al., "Real-time photoacoustic and ultrasound imaging: a simple solution for clinical ultrasound systems with linear arrays," Phys. Med. Biol. 58(1), N1-N12 (2013).

9. B. Yin et al., "Fast photoacoustic imaging system based on 320-element linear transducer array," Phys. Med. Biol. 49(7), 1339-1346 (2004).

10. H. Zafar et al., "Linear-array-based photoacoustic imaging of human microcirculation with a range of high frequency transducer probes," J. Biomed. Opt. 20(5), 051021 (2015).

11. A. Buehler et al., "Video rate optoacoustic tomography of mouse kidney perfusion," Opt. Lett. 35(14), 2475-2477 (2010).

12. J. Xia et al., "Enhancement of photoacoustic tomography by ultrasonic computed tomography based on optical excitation of elements of a fullring transducer array," Opt. Lett. 38(16), 3140-3143 (2013).

13. C. Kim et al., "Handheld array-based photoacoustic probe for guiding needle biopsy of sentinel lymph nodes," J. Biomed. Opt. 15(4), 046010 (2010).

14. V. G. Peters et al., "Optical properties of normal and diseased human breast tissues in the visible and near infrared," Phys. Med. Biol. 35(9), 1317-1334 (1990).

15. Y. Zhou et al., "A phosphorus phthalocyanine formulation with intense absorbance at $1000 \mathrm{~nm}$ for deep optical imaging," Theranostics 6(5), 688-697 (2016).

16. W. F. Xia et al., "Interventional multispectral photoacoustic imaging with a clinical linear array ultrasound probe for guiding nerve blocks," Proc. SPIE 9708, 97080C (2016).

17. J. M. Mari et al., "Interventional multispectral photoacoustic imaging with a clinical ultrasound probe for discriminating nerves and tendons: an ex vivo pilot study," J. Biomed. Opt. 20(11), 110503 (2015).

18. W. Xia et al., "Performance characteristics of an interventional multispectral photoacoustic imaging system for guiding minimally invasive procedures," J. Biomed. Opt. 20(8), 086005 (2015).

19. L. Lin et al., "In vivo deep brain imaging of rats using oral-cavity illuminated photoacoustic computed tomography," J. Biomed. Opt. 20(1), 016019 (2015).

20. P. Kruizinga et al., "Photoacoustic imaging of carotid artery atherosclerosis," J. Biomed. Opt. 19(11), 110504 (2014).

21. D. Piras et al., "Photoacoustic needle: minimally invasive guidance to biopsy," J. Biomed. Opt. 18(7), 070502 (2013).

22. M. A. Lediju Bell et al., "Transurethral light delivery for prostate photoacoustic imaging," J. Biomed. Opt. 20(3), 036002 (2015).

23. H. Assadi, R. Karshafian, and A. Douplik, "Optical scattering properties of intralipid phantom in presence of encapsulated microbubbles," Int. J. Photoenergy 2014, 1-9 (2014).

24. V. N. D. Le et al., "Measurements of extrinsic fluorescence in intralipid and polystyrene microspheres," Biomed. Opt. Express 5(8), 2726-2735 (2014).

25. J. W. Qin and R. F. Lu, "Measurement of the absorption and scattering properties of turbid liquid foods using hyperspectral imaging," Appl. Spectrosc. 61(4), 388-396 (2007). 